\title{
LES AVATARS PROCESSUELS DE LA PENSÉE SYSTÉMIQUE TRANSITIONNELLE VERS UN SYSTÈME RÉSILIENT ET AGILE
}

\author{
Author(s) / Auteur(s) : \\ Amédée Marie ANDRIAMISA-RAMIHONE \\ Doyen de la Faculté des Sciences Sociales \\ Université Catholique de Madagascar \\ amedee.andriamisa@ucm.mg
}

\begin{abstract}
Résumé :
État et situation d'un système postérieurs à un choc, à un traumatisme, la transition "évoque l'idée d'un passage, d'un état à un autre, d'une position à une autre, que ce passage s'effectue graduellement, progressivement et enfin qu'il se passe quelque chose dans l'entre-deux ». C'est un processus qui engendre une interaction continue entre le processus amont et le processus aval, suivant la définition même du processus.

À ce titre, elle établit la liaison entre le choc qui a initié le processus de résistance et le processus de résilience. Le changement qui en résulte affecte la durabilité du « système télescopé et traumatisé (STT)».

C'est à partir de ces divers éléments de définition que les enjeux et les conditions de vie (ou de sur-vie) de la transition sont précisés à l'aide de différents exemples et modèles en lien avec la systémique et ses applications dans le domaine du changement.

Forgeant un système dynamique complexe, non-linéaire et chaotique, la transition avec la résilience (réactive et pro-active) induit le changement qui à son tour crée l'incertitude. Une adaptation s'impose pour comprendre le futur qui vient et qui est imprédictible dans un monde VICA! Le processus du changement en U semble univoque et traduit paradoxalement une voie toute tracée, ce qui n'est pas le cas du processus en Wn ou en L! Loi de Murphy oblige!

La pensée systémique semble se présenter en panacée de par la durabilité des systèmes complexes, mais un concept plus fécond lui vient en aide, suivant la dialogique morinienne pour y adjoindre le concept de «système résilient et agile »; marquant le passage de STT à SRA!

Telle est la conviction de cet article qui essaie d'innover dans le domaine de la science politique, notamment en politiques publiques elles-mêmes systèmes complexes...
\end{abstract}

Keywords / Mots-clés :

transition, résilience organisationnelle, processus, changement, système télescopé et traumatisé, système résilient et agile

\section{CONTEXTE INTRODUCTIF.}

Il est révolu le temps où le monde était perçu comme un "long fleuve tranquille", sans heurts ni fortes secousses, avec moins de population, moins d'informations, moins de produits à consommer, moins de flux, etc... ! Le contexte a bel et bien évolué et les temps ont changé ! Et l'on est actuellement en présence d'un bouleversement social et sociétal enragé et frénétique : involutions, évolutions et révolutions ont modifié la face du monde, ont remodelé la carte du monde ! De nouveaux besoins, de nouvelles possibilités se réifient.

Explosion des flux et des réseaux, mutations technologiques, économie de marché, montée du chômage, inégalités sociales, enchainement des crises financières, primauté des entreprises et multinationales au détriment des politiques, etc... sont les facettes dérangeantes d'une mondialisation, heureuse ou non, d'une globalisation inégalitaire ! Un monde en et de turbulence, un monde agoantagoniste apparait !

"Un monde « de turbulences », chaotique, incertain et instable, mais aussi riche en opportunités et porteur de promesses » titraient les Rencontres économiques d'Aix, au vu de la crise financière de 2008, des Printemps arabes, de la crise grecque ayant failli aboutir à un Grexit, et maintenant le Brexit 
dans tous ses états. "Le contexte de compétition générale crée de l'instabilité durable puisqu'aucune force stabilisatrice n'émerge [...]. Et ce n'est pas fini ! Les turbulences que nous connaissons vont perdurer dans tous les domaines. » estimait Hubert Vedrine, sous les plumes de Caroline Castets. Et ces turbulences essentiellement macro-économiques ne sont pas sans bouleverser ou impacter les équilibres politiques et démocratiques : le concept de «broken political system » de Suzanne Berger, professeur au MIT, résume d'une manière générale l'ambiance délétère et d'incertitude qui prévaut, eu égard à ces turbulences.

Et ces turbulences ne sont pas sans impact sur l'ordre mondial. Pour Paul Valéry, l'humain dans son anthropocène ne cesse de d'osciller entre ordre et désordre (Moreau-Defarges, 2008) :

\begin{abstract}
le premier l'ennuie et le second l'effraie. Dès qu'il a l'un, il s'en sent prisonnier et cherche l'autre. Il n'existe pas - heureusement - d'ordre définitif, parfait ; tout ordre réel est partiel, inachevé, tolérant ou supportant des zones de désordre. A l'inverse, aucun désordre n'est total ; tout chaos humain notamment les guerres [donc les crises, dirons-nous] suscite des ordres spontanés, sauvages. Si un ordre parfait équivalait à la mort, un désordre total est impossible, les hommes dans les pires catastrophes, produisant toujours des règles, des codes ;
\end{abstract}

Quant aux militaires auditeurs de la $68^{\circ}$ session du Centre des Hautes Études Militaires (CHEMFrance), ils ont intentionnellement intitulé le Cahier de la Revue de Défense Nationale (RDN) sous le titre «Un monde en turbulence » avec trois thématiques, à savoir (i) un contexte géopolitique instable, puis (ii) des défis stratégiques renouvelés, et enfin (iii) l'humain au cœur de la transformation. Le préfaceur étoilé Jean Christophe Bechon, directeur du CHEM, introduit le Cahier en ces termes à propos de cette turbulence :

\begin{abstract}
Fondamentalement, nos démocraties occidentales font face au défi gigantesque du deuxième âge de la mondialisation, du partage des richesses, de la maitrise des flux et de la violence, à un moment où une forme de désinhibition et de déstructuration semble en passe de l'emporter. L'exponentielle des bouleversements frappe par son intensité, tout comme les cloisonnements transparents que le numérique favorise ou la norme qui souvent contraint.
\end{abstract}

Et dans ce tumulte turbulent présageant un retournement, pis encore un effondrement, dans ce bouillonnement déroutant, dans cette frénésie inhibitrice de toute grille de lecture rationnelle, les systèmes (et sous-systèmes) de toute nature se bousculent et se fractalisent par isomorphisme ou homomorphisme, se heurtent mutuellement à de niveau de létalité différent, se succèdent dans le temps et de par leur interdépendance, affectant ainsi leurs propriétés synchronique et/ou diachronique. Imprévisibilité, imprédictibilité, incertitude sont au rendez-vous !

De ce qui précède, la situation présente semble alors s'apparenter à une situation de crise. Et une crise - polymorphe de surcroit - n'est pas à exclure : elle peut être (i) sociale, (ii) politique, (iii) économique, (iv) financière, (v) écologique, (vi) démographique, (vii) humanitaire et humaniste, (viii) culturelle, etc... Une crise systémique est le pendant de cette effervescence irresponsable, une sorte de "dérapage incontrôlé" (ou plutôt qu'on essaie de contrôler à coups de conférences et de sommets) mais qui va se terminer par une "sortie de route" dans ce "monde fini", si l'on se repaît dans l'inaction! (Je crois que je vais voter pour ce loup, ça fera réflechir le berger).

Dans l'éditorial de la Revue Acta europeana systemica, Damien Claeys, professeur à l'Université catholique de Louvain, précise que « les crises ne sont plus considérées comme de simples adaptations de systèmes pré-crises, mais elles sont les sources potentielles de l'émergence de structures nouvelles. Ces dernières sont générées par l'intégration de nouvelles connaissances et technologies, l'amélioration de la gestion des ressources, la redéfinition d'un équilibre sociopolitique » (Claeys \& Minati, 2018, p.2).

Synonyme de tension entre deux forces paradoxales, la crise est annonciatrice de disruption : elle est déjà un changement. Plus subtile encore est l'idée selon laquelle la crise n'est pas seulement les prémisses d'une transition : elle est déjà une transition vers un nouvel équilibre! Il s'agit de la métastabilité d'un système. 
Fatalement, les crises encourues ou à venir interpellent un changement, un changement dans la façon de penser des acteurs et dans les actes eux-mêmes ! Janine Renier associe la crise à la méta-morphose ${ }^{1}$ pouvant éventuellement déboucher sur le "Grande transition". Etape incontournable du processus de changement, la crise revèle une situation obsidionale dans laquelle la tension, la peur, le déséquilibre, l'incertitude, l'inattendu se sont constitués comme facteur de rupture de l'équilibre pré-existant ! Instinctivement, l'espèce humaine dans son anthropocène réagit et essaie de survivre en opérant un choix, en portant un jugement contingent, en prenant une décision importante, ce qui nous ramène à la définition etymologique grecque du vocable "krisis". Le système est ramené "suite à cet instinct de survie" à son état antérieur d'équilibre initial et par un mécanisme d'auto-régulation permanent : le principe d'homéostasie !

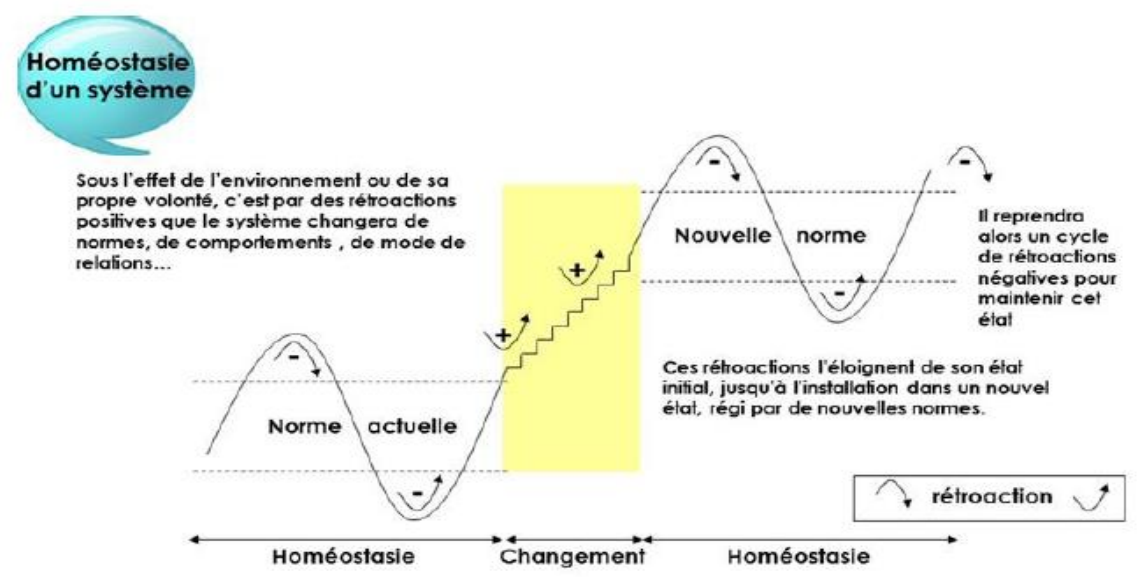

Figure 1 -Homéostasie et Changement (Janine Renier, 2018 - p. 289)

Dès lors, pléthore de questions viennent interpeller la pensée systémique en quête d'outils pratiques de modélisation.

Comment appréhender la pensée systémique dans ce monde là ? Est-il possible et utile d'anticiper ? Quelle attitude, quelle posture intellectuelle adopter ? Etre pro-actif ou rester réactif ?

On ne sait pas comme se comporter, comment se conduire ni répondre à cette effervescence turbulente du monde. On ne maîtrise pas les aléas, puis on subit les contre-coups du changement qui en résulte.

Comment raisonner en terme de système ? Ce dernier se prête comme l'outil par excellence permettant d'analyser en amont le changement.

Méthodologiquement, il sera procédé à la réification du système pour évaluer son comportement, ses attitudes.

(i) En lui faisant subir virtuellement un choc, un traumatisme, une crise pour voir ce que cela donne.

(ii) En manipulant le système dans son environnement (éco-système)

(iii)En inventoriant et en analysant ses différents états possibles dans le temps, pour envisager un autre système plutôt que de persister dans et vers une optimisation des processus.

Cette réification se déclinera alors séquentiellement en termes de processus selon (i) les conditions initiales du système, puis (ii) quand il sera télescopé et traumatisé pendant cette phase de transition, et finalement (iii) quand on assista à une résilience oragnisationnelle du système qui innovera dans l'agilité. Ces perspectives se déroulent et se pratiquent dans le cadre des politiques publiques qui sont des systèmes complexes, ne se lasse-t-on pas de mentionner !

\footnotetext{
${ }^{1}$ Acta Europeana Systemica n ${ }^{\circ} 8$
} 


\title{
LES PRINCIPAUX CONCEPTS DU SYSTEME
}

\author{
Selon l'état de l'art.
}

Jamais un mot n'a été aussi galvaudé que la mondialisation. Il a été assimilé à beucoup de concepts, à tort ou à raison, et les sens véritables en changent selon les auteurs, leurs penchants, leurs profils idéologiques, etc...bref selon les contingences. Et le mot système subit les mêmes travers que le vocable mondialisation. Et la vulgate de s'en servir au détriment de la doxa qui a tenté d'endiguer cette vague par une précision du concept. Les définitions esquissées en sont multiples, et il n'est pas redondant d'en reprendre quelques-unes ici afin de cerner avec précision le sens affecté à cet article.

Ainsi, dans son état normal, dans ses conditions initiales avant d'être l'objet ou le sujet de quelque événement, le système se définit comme "une totalité organisée, faite d'éléments solidaires ne pouvant être définis que les uns par rapport aux autres en fonction de leur place dans cette totalité" selon Ferdinand de Saussure. (Durand, 1979). Celle donnée par Ludwig von Bertalanffy la pose comme "un ensemble d'unités en interrelations mutuelles", alors que J. Lesourne postule que c'est "ensemble d'éléments liés par un ensemble de relations".

Une autre vague de trois autres auteurs la définit successivement comme "un ensemble d'éléments en interactions dynamique, organisés en fonction d'un but" (Rosnay, 1975), puis comme "une unité globale organisée d'interelations entre éléments, actions ou individus." (Morin, 2004), et enfin J. Ladrière selon lequel c'est "un objet complexe, formé de composants distincts reliés entre eux par un certain nombre de relations."

Les quatre concepts fondamentaux reconnus par les uns et par les autres au système ainsi défini se rapportent à (i) l'interaction qui évite la simple causalité linéaire pour privilégier la mutualité, la double causalité ; (ii) la globalité stipulant que le système composé d'éléments n'est pas la somme des éléments, est irréductible à ses éléments (Bertalanffy, 1993) et est à la fois plus et moins que la somme des parties (Morin, op. cit.), et conséquemment, développe cette propriété d'émergence que les parties $\mathrm{du}$ tout n'ont pas ; (iii) l'organisation qui revêt une double signification, à la fois structurelle et fonctionnelle ; et enfin (iv) la complexité dûe à trois faits, à savoir 1/la composition du système : nombre élevé puis diversité des caractéristiques et surtout la nature des liaisons entre les différentes parties du système; puis 2/l'incertitude inhérente à l'nvironnement du système ; et enfin 3/l'introuvable et incestueuse relation entre l'ordre et le désordre.

Au-delà de ces caractérisations définitionnelles du système, appréhendons-le suivant le triptyque "environnement externe-attributs internes-le temps".

\section{De l'environnement externe du système}

Vis-à-vis de son environnement, le système peut être - dans l'absolu - ouvert ou fermé ou isolé ! C'est presque un non-sens dans la mesure où "un système ne saurait être entièrement ouvert, c'est-à-dire sans frontière marquant ses limites $[\ldots]$ : il se confondrait indistinctement avec [l'environnement] et ne pourrait être identifié. Toutefois, il ne saurait davantage être entièrement fermé, car tous les systèmes [...] dépendent pour leur survie de leurs échanges avec ce même environnement. En fait, [...] tout système est sélectivement ouvert ou fermé selon la nature des intrants et des extrants énergétiques, matériels ou informatifs - dont il dépend pour son existence." (François, 1998). Pour plus de consensus, Bernard Walliser le nomme "un système quasi-isolé" (1977).

En outre, un système peut être dans un état d'équilibre et de stabilité, d'abord dans la mesure où il reste dans cet état en l'absence de modifications de l'entrée, et puis lorsqu'on modifie soit les conditions aux limites du système (stabilité asymptotique), soit les fonctions de définition du système (stabilité structurelle) (Walliser, 1977). Pour aller plus loin, ajoutons le concept de "métastabilité systémique" qui suppose la propriété [première] d'un état d'apparence stable, mais qu'une perturbation peut faire aller rapidement vers un état [second] encore plus stable. 


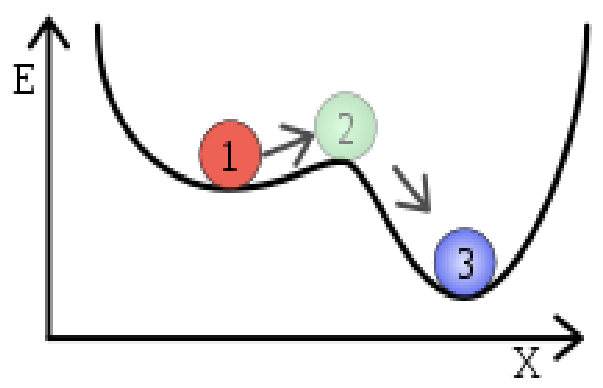

Figure 2 - Métastabilité systémique. États d'un système métastable : L'état 1 paraît stable, mais une perturbation suffisante pour dépasser l'état 2 conduira à l'état 3, plus stable que l'état 1 (qui de fait n'est que métastable). Source : https://fr.wikipedia.org/wiki/M\%C3\%/A9tastabilit\%C3\%A9

Et la dernière propriété externe du système qui nous interesse est son adaptation. Il est qualifié "adaptatif" s'il peut - tel un caméleon - adopter [positivement] un comportement favorable à ses finalités, tenant compte de l'environnement (Walliser, p. 37). Dans le cas négatif, le système peut opter pour "battre en retraite" selon l'expression militaire consacrée, c'est-à-dire quitter le champ, quitter l'environnement qui ne lui est pas, qui ne lui a pas été favorable pour en opter pour un autre plus "positif". Les concepts connexes à l'adaptation sont l'homéostasie qui est la tendance naturelle du système à se maintenir dans son état initial par un mécanisme d'auto-régulation (Cannon, 1932, p. 22), et l'homéorhésie qui est l'adaptation d'un système au maintien d'un processus constant (Waddington, 1975, p. 221-223). Un concept plus fécond et qui rejoint celui cité supra et se rapportant au tout, à la totalité, vient renforcer le dispositif d'adaptation et de stabilité, à savoir l'homéotélie ! Du grec homoios (même) et telos (but), ce vocable s'applique à tous les systèmes naturels, et il prône le "maintien du tout" des processus du vivant (Bertalanffy, 1962, p. 123).

\section{Des caractéristiques internes du système}

Indubitablement, un système se concoit - dans l'absolu - comme un tout avec plusieurs éléments que l'on peut assimiler à des sous-systèmes à leur tour. Il reçoit dès lors le qualificatif de système quasidécomposable caractérisé par des interrelations entre les sous-systèmes, par l'intermédiaire des entrées et sorties de chacun de ces sous-systèmes, et aussi avec l'environnement qui les entoure. Dès lors, il peut être qualifié de soit un hyposystème, c'est-à-dire un système avec seulement quelques soussystèmes sauvegardés, épargnés, mais avec toutes leurs relations ; soit un infrasystème si tous les soussystèmes sont gardés et conservés, mais avec quelques relations seulement. Cette situation nous sera rappelée quand on abordera la partie où le "système est télescopé et traumatisé" !

Les autres propriétés internes du système telles que l'équilibre, la stabilité sont les mêmes, identiques à celles exposées supra concernant l'environnement externe, et ne seront plus développées céans. Toutefois, il est opportun de signaler qu'un système peut être composé de sous-systèmes plus ou moins interdépendants entre eux, qu'ils soient intégralement isolés, peu ou prou, et donc sont qualifiés d'agrégat, ou avec des éléments indiscernables, donc intégrats. (Walliser, 1977).

\section{De la temporalité du système}

Il est notoire et compréhensible qu'un système ne peut pas être "a-temporel” : il n'échappe pas au temps, et lui est assujetti ! Mais cela signifie aussi que le système connait une période - même brève au cours de laquelle elle semble s'en échapper : c'est la période d'invariance, qu'on dénomme la durée de vie du système ! Il conserve et sauvegarde pendant ce laps de temps (très variable) les caractéristiques de son maintien de sa survie.

La seconde caractéristique se rapportant à cette temporalité du système concerne la continuité ou la discontinuité. Un système ne peut pas "exister à flux tendu" : il lui est impossible de fonctionner, d'être et d'exister de la même manière, avec le même tonus, avec la même vitesse, avec les mêmes processus pendant une période observable. Des périodes de rupture, de baisse de tension, ou aussi de performance, de génie, etc...- fussent -elles brèves, ponctuelles et saccadées - sont inévitables : 
continuité et discontinuité s'alternent, à des phases différentes certes, et rythment par saccades ou avec fluidité le tempo de tout système.

Pour clore sur le concept de système, empruntons à la psychologie sa représentation de l'emboitement des niveaux possibles de systèmes (Caublot, M. et Blicharski, T. 2016, pp. 103-120), sans avoir la possibilité dans cet article d'aller au plus profond en étudiant point par point les différents composants de chaque niveau (6 niveaux en tout) :

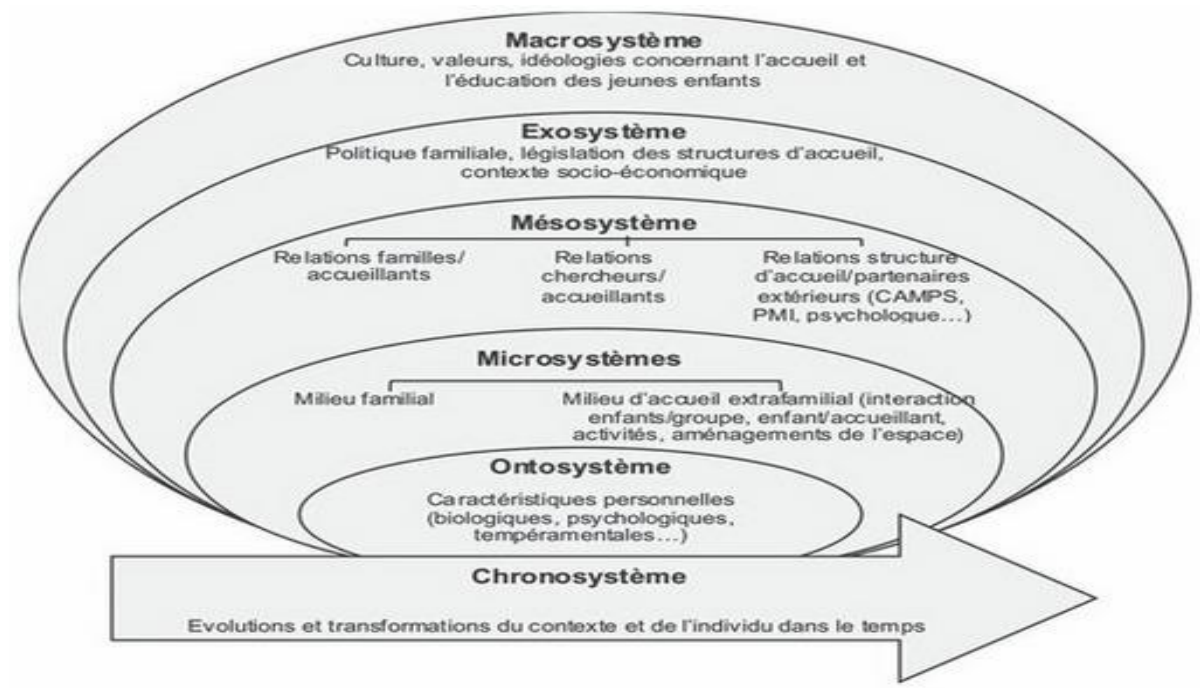

Figure 3 - Représentation schématique du modèle écosystémique de la qualité, adapté de Bigras et Japel, 2007 ; Lemay et Bigras, 2012.

Pour terminer, cet état de l'art disséquant les divers points de vue d'éminents chercheurs a été intentionellement intitulé "les conditions initiales" rapportant les "états normaux, naturels" de tout système, car il sera abordé infra le cas de système dynamique, chaotique.... "sensible aux conditions initiales" (SCI) lors du choc, lors de la collision.

\section{QUAND LE SYSTEME EST TÉLESCOPÉ ET TRAUMATISÉ}

Retour d'expérience

\section{Le pendule de Newton et le Chat de Scrödinger}

La pertinence scientifique invite à une définition des concepts usités, et il en sera de même pour le télescopage. Le choix n'en est pas anodin dans la mesure où le système qui subit un choc venant de son environnement ( une crise, une défaillance, une évolution, etc. ) peut retrouver a posterori son état initial, du moins certaines de ses caractéristiques sans pour autant disparaître du champ, de par ses aptitudes d'auto-organisation. Le télescopage n'est pas létal ! Tout juste quelques traumatismes, graves ou pas selon l'intensité du choc et selon le mouvement imprimé !

Le pendule de Newton est un corollaire qui pourrait représenter le choc, sans qu'il soit nécessaire d'approfondir et d'aller jusqu'au choc mécanique imprimé, élastique ou inélastique ${ }^{2}$ ! En effet, on parle de choc (ou collision) lorsque "deux points matériels (ou particules) initialement isolés l'un de l'autre entre en interaction pendant une durée suffisamment courte", car tout choc conserve la quantité du mouvement ! Le système télescopé par un choc venant de son environnement reproduirait-

\footnotetext{
${ }^{2}$ Un choc est élastique si ce choc conserve l'énergie cinétique totale du système. Il n'y a aucune modification de l'état interne des deux corps en collision; et le choc est inélastique s'il ne conserve pas l'énergie cinétique totale du système. Disponible à l'adresse www.ensa.ac.ma/docs/pedagogie/bouhyahia/CH6.pdf

${ }^{3}$ Ibid.
} 
il le même scénario ? En étant traumatisé pour un court instant, certes, mais traumatisé toujours ? Ou encore un système burn out !

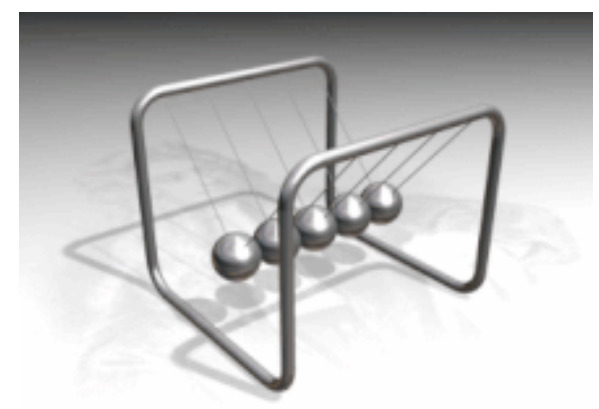

Figure 4.1 - Le pendule de Newton au repos
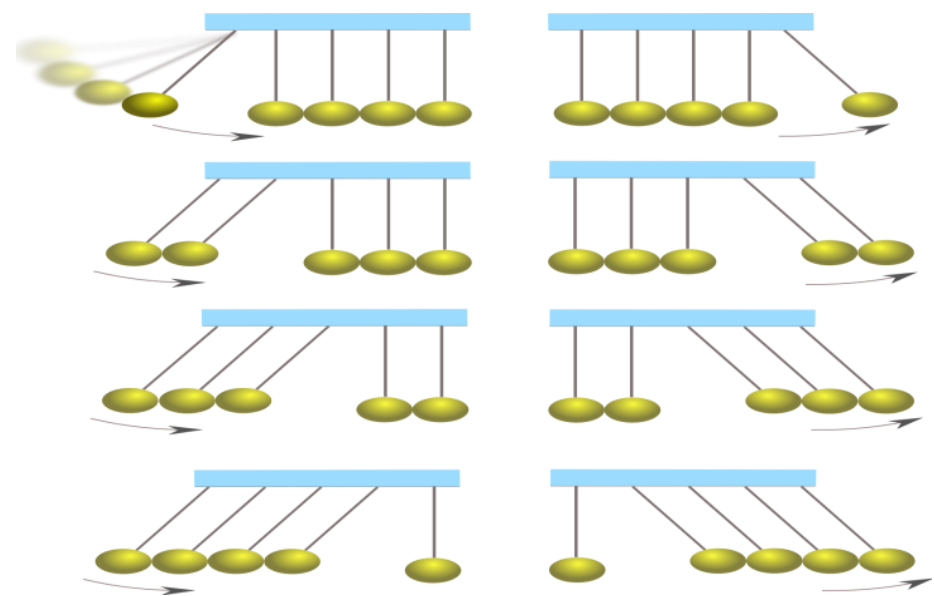

Figure 4.2 - Le pendule en mouvement, alternativement par boule : 1 , puis 2 , puis 3 et enfin 4

Figure 4 - Le pendule de Newton. Source : https://fr.wikipedia.org/wiki/Pendule_de_Newton.

Ceci dit, faisons quelques pas en arrière en nous référant à Aristote. Selon la bonne vieille logique aristotélicienne, qualifiée également de logique classique, trois axiomes prédominent et définissent la logique :

- L'axiome d'identité : A est A

- L'axiome de non-contradiction : A n'est pas non-A

- L'axiome du tiers exclu : il n'existe pas un troisième terme qui soit à la fois A et non-A.

Ainsi, il appert qu'un système ne peut pas être un non-système, et partant, s'il est dans un état donné, il ne peut pas l'être autrement, ou dans un autre état. Dans son évolution dans le temps, et ayant été télescopé, notre système n'est plus alors dans son état initial, délaissant ses "propriéts synchroniques" et acquérant ainsi celles diachroniques.

Par contre, le Chat de Schrödinger nous apporte une ouverture, une vision autre que cette bonne vielle logique classique. En effet, il s'agit d'une expérience de pensée ${ }^{4}$ virtuelle (elle n'a jamais été effectuée physiquement) imaginée par l'autrichien Erwin Schrödinger

Il a donc imaginé l'expérience suivante : il enferme son chat dans une boite close, contenant un dispositif qui tue l'animal dès qu'il détecte la désintégration d'un atome d'un corps radioactif. De l'extérieur, on ne peut pas savoir ce qui se passe dans la boite.

En clair : le chat dans la boite peut vivre ou mourir, sans que l'on sache ce qui se passe depuis l'extérieur.

\footnotetext{
${ }^{4}$ Une expérience de pensée, c'est tenter de résoudre un problème en utilisant uniquement son imagination. C'est se poser la question : "que se passerait-il si.....?
} 


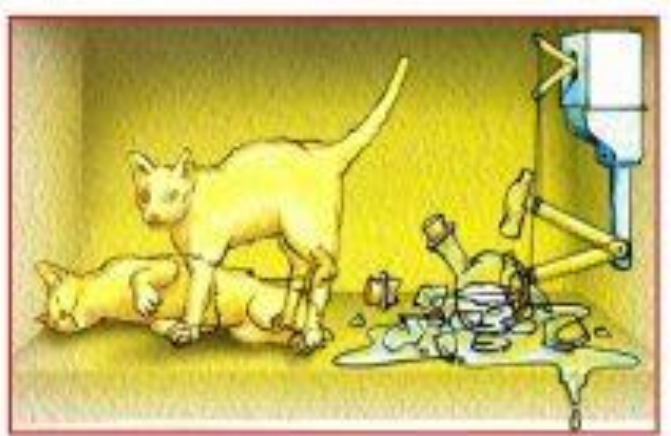

Figure 5 - Dessin de l'expérience de Schrödinger.

Source : https://fr.wikipedia.org/wiki/Chat_de_Schrödinger.

Voilà ce que dit la logique classique, mais la logique quantique modife ce deuxième axiome de la logique classique avec l'axiome de "non-contradiction".

\begin{abstract}
Schrödinger s'imagine donc à côté de cette boite totalement fermée et se dit :
Je n'ai aucun moyen de savoir si le mécanisme s'est déclenché. Si mon chat était un objet quantique, je dirais qu'il est à la fois mort ET vivant car selon la théorie de la superposition quantique, c'est tout à fait possible.

La seule manière pour lui de savoir si son chat est mort ou vivant, c'est d'ouvrir la boite et de constater. Lorsqu'Erwin ouvrira la boite, on parlera de décohérence quantique. La situation ambiguë ("mort ou vivant ?") se résout instantanément car une fois la boite ouverte, le minou n'est plus "ou mort ou vivant", il est clairement soit l'un, soit l'autre.
\end{abstract}

Certains systèmes quantiques comme les atomes, les photons ou les spins peuvent adopter deux états simultanément. Ils sont aussi appelés "Chats de Schrödinger". [...] Dès que l'objet quantique superposé dans deux états interagit avec son environnement, qu'il s'agisse d'atomes, de lumière ou de chaleur, la superposition cesse au bout d'un temps très court appelé "temps de décohérence". Plus l'interaction est importante, plus ce temps est court ${ }^{5}$.

Quelque soit le cas de figure qui se présente ou qui pourrait se présenter, superposition des deux états du système (sauf ou traumatisé) ou un seul état traumatisé suite au télescopage, le résultat est là : son état initial a évolué vers un état second, au propre comme au figuré ! Il y a un changement morphologique du système, fût-ce en un temps très court !

\title{
Le Processus en $U$, puis en $W$
}

Un autre concept en vogue actuellement que le changement. Mais changement n'est pas transition ! Nous reviendrons sur le concept du changement, après avoir expliqué celui de la transition.

\section{La transition}

Par définition, elle est le passage d'un état d'équilibre homéostasique à un autre, selon un processus qui suit des périodes de stabilité relative et des périodes de transformations structurelles (Loorbach, 2007) traversant plusieurs phases (multiphase) : phase de pré-développement, puis phase de décollage, et phase de percée, et enfin phase de stabilisation, selon Kemp et Rotman (2004).

Dans la même logique ordinale, ces phase sont assimilées (i) au choc, puis (ii) à la résistance, et (iii) à l'ouverture, et enfin (iv) à l'implication.

Au-delà de cette conceptualisation définitionnelle de la transition, «Importante également est l'idée qu'un processus de transition est un phénomène à la fois micro - et macro-sociologique, qu'il est fait d'une multitude de naissances spontanées, de développements locaux dont beaucoup sont éphémères mais qui s'additionnent peu à peu et dont les effets convergent sous forme de structures globales

\footnotetext{
${ }^{5}$ Disponible à l'adresse https://toutestquantique.fr/superposition/
} 
nouvelles qui entreront en conflit avec la reproduction des structures globales du système dominant » (Godelier, ????).

Outre-mozambique (c'est-à-dire au Rwanda, par rapport à Madagascar), la façon d'appréhender la transition se décline comme suit, selon Terry Irvin (Carnegie Mellon University) :

Change is the physical end-state of a transformation; it is the new situation and/or circumstance that is observable and measurable.

Transition is the psychological process of making something different; it occurs within the person(s) experiencing the change. The transition process is an internal, esoteric experience. Therefore it is not directly observable and is extremely difficult, if not impossible, to measure or to track.

The major difference between change and transition is that transition is not a physical outcome, but rather a psychological state of mind: the letting go of the old reality in order to fully and effectively realise the benefits of the new reality 6 .

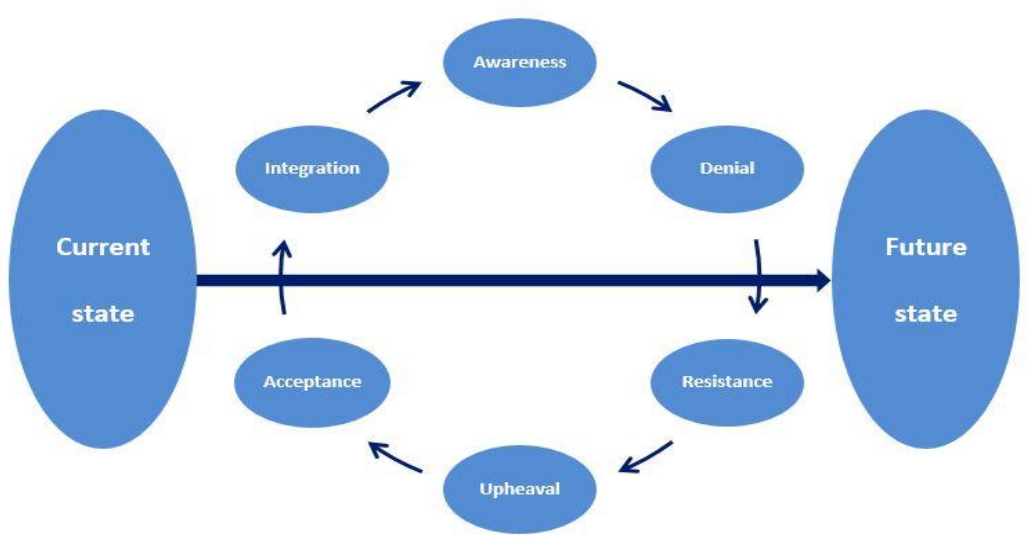

Figure 6 - Change and Transition leadership (Terry Irvin, 2017)

Pour compléter le tableau synoptique afférent à la transition, l'apport de William Bridges diffère de celui de Kemp et Rotman en ce sens qu'il a "phasé" la transition en étapes, à savoir 1/une fin, puis $2 /$ Zone neutre, et enfin 3/un nouveau départ.

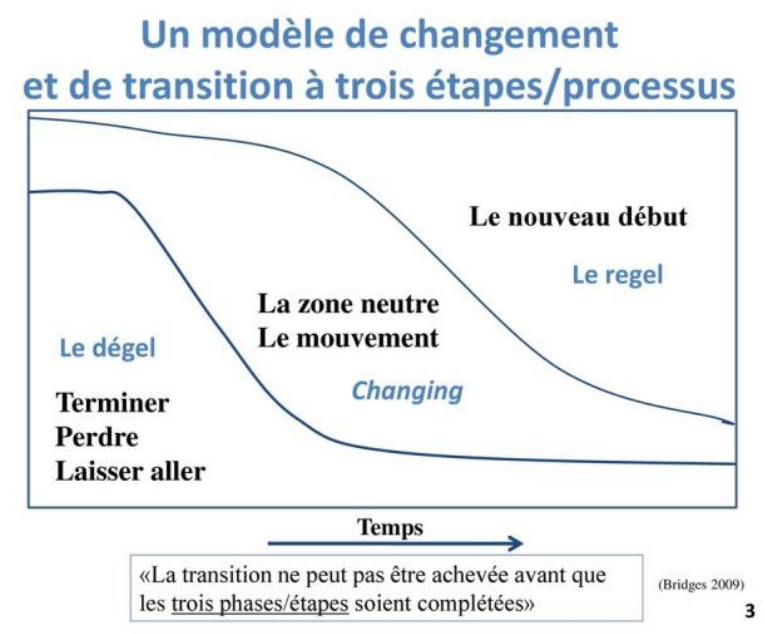

Figure 7 - La transition en 3 étapes (William Bridges, 2009)

Plus explicite, cette transition est déclinée en une nouvelle mouture mettant en évidence le lien entre le changement et la transition

\footnotetext{
${ }^{6}$ Disponible à l'adresse https://www.tcii.co.uk/2017/09/13/change-transition-leadership/
} 


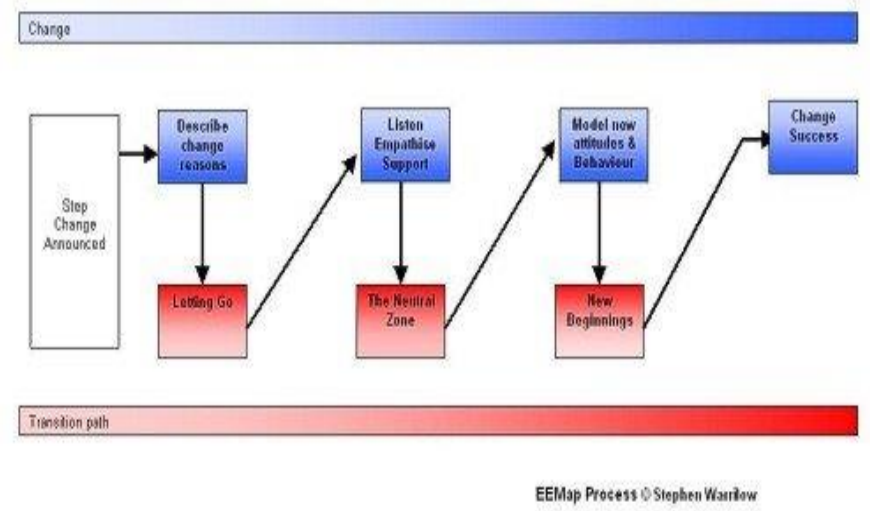

Figure 8 - La transition (plus explicite) de William Bridges, 2009

Postérieure ou également inhérente au changement, la transition est ainsi un processus interne d'adaptation psychologique à une nouvelle réalité. Les répercussions de la transition ne sont pas nécessairement liées à l'importance apparente du changement qui l'a déclenchée. Toutes les transitions commencent par (i) la fin de quelque chose, (ii) se déroulent pendant la zone neutre et (iii) se terminent par un nouveau début.

Une fin : Chaque transition commence par une fin, exprimée par un sentiment de perte, de déni, de confusion, de désir de changement mais freiné, temporisé et entrant en conflit avec la résistance à «lâcher prise ».

Zone neutre : La zone neutre est une période trouble de recherche, de confusion, de penser à la fuite, de questionnements aussi, et surtout de doute sur le futur tout en restant nostalgique du passé...

Nouveau départ : le bond et le rebond pour une "nouvelle aventure" !

De ce qui précède, l'on atteint l'apothéose en parlant de la Grande Transition : qu'est-ce que c'est ?

«Lancée initialement par le Global Scenario Group (Raskin et al. 2002), cette notion a été adoptée par d'autres acteurs par la suite, par exemple par la New Economics Foundation au Royaume-Uni (Spratt et al. 2010). Elle offre un cadre conceptuel pour penser une économie et une société mondiales soutenables, et développer les stratégies nécessaires à leur avènement ${ }^{7} \gg$.

L'idée principale de la Grande Transition est d'abord de faire cesser cette folie qu'est "La dégradation progressive des écosystèmes de la planète, qui fournissent la nourriture et l'eau potable nécessaires à la vie humaine, [et qui] est directement liée à l'augmentation rapide de la demande en ressources naturelles d'une partie de la population mondiale dont la richesse matérielle et le pouvoir d'achat connaissent une croissance sans précédent. En parallèle, le fossé entre riches et pauvres se creuse dans de nombreux pays, et des milliards de personnes continuent à vivre au-dessous du seuil de pauvreté ${ }^{8} »$, en se posant ensuite les bonnes questions de réflexion, pour enfin passer à un nouveau système, car le monde poursuit un développement profondément insoutenable! «You never change things by fighting the existing reality. To change something, build a new model that makes the existing model obsolete », disait Buckminster Fuller.

Toujours à la recherche du Graal de la pensée systémique qui aiderait à réduire la vulnérabilité du système pour un développement soutenable, les systémiciens ne rejetteraient pas les principes et les prémisses esquissées pour ce faire.

\footnotetext{
${ }^{7}$ Rapport de Michael Narberhaus en collaboration avec Christina Ashford, Maike Buhr, Friederike Hanisch, Kerem Şengün et Burcu Tunçer, Traduit de l'anglais par Emilie L'Hote, revu par Wojtek Kalinowski, Titre original : « Effective change strategies for the Great Transition. Five leverage points for civil society organisations ».Stratégies pour la Grande Transition, Cinq leviers de changement pour les Organisations de la Société Civile, mars 2011, s.1.

${ }^{8}$ ibid.
} 
Ces prémisses sont déclinées comme suit :

1. Le changement doit être un changement systémique

2. La transformation nécessaire des valeurs culturelles

3. Une économie au-delà de la croissance matérielle et du consumérisme est souhaitable, possible et nécessaire

4. Le PIB n'est pas forcément la clef du système économique

5. Il y a une vie après le consumérisme

Et les principes s'énumèrent de la façon suivante :

1. Principe de bien-être social

2. Principe de suffisance

3. Principe d'efficacité

4. Principe d'éco-compatibilité

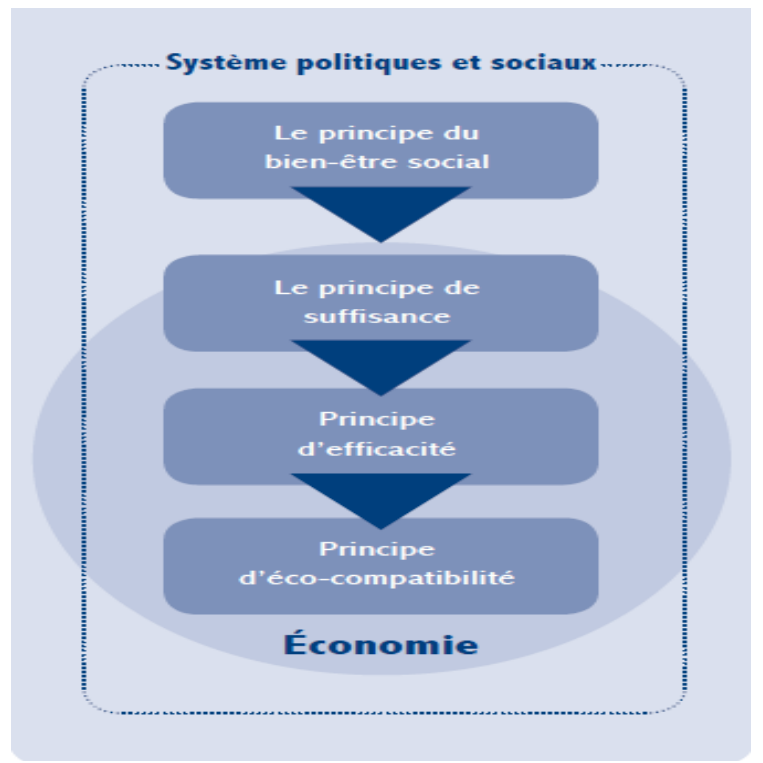

Figure 9 - Hiérarchie des principes.

Source : La Grande Transition, Cinq leviers de changement pour les Organisations de la Société Civile, page 14

Déclinée et présentée de la sorte, la Grande Transition se perçoit comme un plan de transition.

En résumé, le plan de transition correspond à la programmation des actions d'accompagnement du changement. Pour les processus et structures, les impacts relevés dans l'étude d'impacts sont traités avec la proposition d'actions d'accompagnement. Les actions sont ensuite mises en œuvre pendant le projet auprès des intéressés (Autissier et Moutot, 2013 - Chapitre VII, L'accompagnement du changement)

\section{Le changement}

S'il en est ainsi de la transition, qu'est-ce que le changement alors ? Outre l'explication pratique et l'exemple de changement supra, une définition plus académique du changement s'impose : c'est le "passage d'un état à un autre, processus de transformation durable et observable qui impacte, de manière substantielle ou marginale, le fonctionnement d'une organisation et/ou d'un individu ${ }^{9} »$ !

Terme polysémique par excellence, le changement est souvent connoté négativement, car associé à une rupture de l'environnement actuel et du futur imaginé ou souhaité qui vient.

Le changement est une rupture dans le fonctionnement lorsque les éléments suivants sont transformés (Autissier et Moutot, 2013, ibid) :

\footnotetext{
${ }^{9}$ Tapia, 2001/2002, page 7
} 
- Les pratiques : manières de faire

- Les conditions de travail : environnement matériel

- Les outils : informatiques et de gestion

- L'organisation : les zones de pouvoir et les délimitations fonctionnelles

- Le métier : les savoir-faire de l'organisation

- La stratégie : les finalités collectives poursuivies et envisagées

- La culture : le système de valeur

Selon la nature, la durée et l'intensité de ce passage, on parlera d'évolution, de révolution, de transformation, de métamorphose, de modification, de mutation de régression (ie de transformation profonde et durable).

\section{Typologie du changement :}

Tableau 1 - Principales caractéristiques du changement - Typologie des situations de changement qui influencent la démarche

\begin{tabular}{|c|c|c|}
\hline & Changement Imposé & Changement Volontaire \\
\hline $\begin{array}{c}\text { Changement } \\
\text { Progressif }\end{array}$ & $\begin{array}{l}\text { Changement prescrit } \\
\text { Réponse à des contraintes de l'environnement } \\
\text { (réglementaire, technologique, etc.), à une } \\
\text { nouvelle stratégie } \\
\text { Ex : la monnaie euro, les } 35 \text { heures }\end{array}$ & $\begin{array}{l}\text { Changement construit } \\
\text { Évolutions des comportements, des pratiques, } \\
\text { puis de l'organisation qui amènent à changer } \\
\text { les manières dont les acteurs se représentent } \\
\text { leur entreprise } \\
\text { Ex: Culture client, qualité, processus }\end{array}$ \\
\hline Changement Brutal & $\begin{array}{c}\text { Changement de crise } \\
\text { Solution à une injonction politique ou } \\
\text { règlementaire urgente, à un dysfonctionnement } \\
\text { grave } \\
\text { Ex : Accident, grève, plaintes ou doléances de } \\
\text { clients }\end{array}$ & $\begin{array}{c}\text { Changement adaptatif } \\
\text { Ex: Transformations structurelles puis des } \\
\text { pratiques, des comportements et enfin de } \\
\text { l'organisation } \\
\text { Nouvel outil informatique, compétences } \\
\text { techniques nouvelles }\end{array}$ \\
\hline
\end{tabular}

Source : adapté de Autissier et Moutot (2013)

Le changement le plus significatif et le plus marquant a été celui concrétisé par la courbe de Kurt Lewin, puis la courbe de deuil de Kubler-Ross, et enfin la théorie U de Otto Scharmer. Rapportées à un changement systémique, postérieur à une crise dans le cas de cet article, ces courbes retracent d'une manière générale 3 moments forts, à savoir (i) l'éclatement, l'implosion, la chute, le déclin, etc. puis (ii) la phase de transition proprement dite, la phase de résistance du système, et enfin (iii) la renaissance, le retour, la montée en puissance, la "remontada", bref la résilience.

Dans l'absolu, le STT épouse le tempo des "modèles applicatifs de courbes classiques" citées supra et qui sont représentées successivement comme suit : 
Les 3 niveaux dans la conduite du changement (Kurt Lewin, 1947)

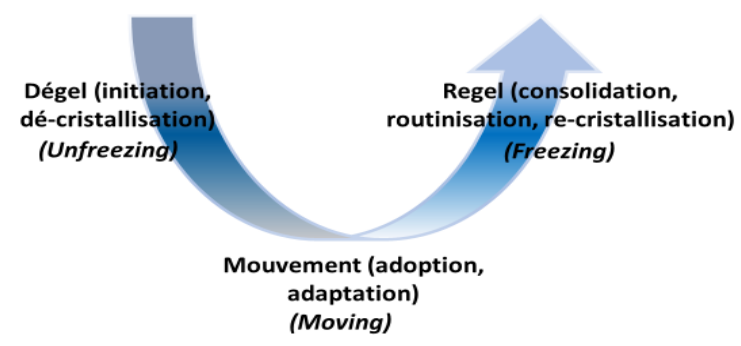

Figure 10 - La courbe en 3 étapes (Kurt Lewin, 1947)

\section{La courbe du deuil ou courbe du changement de Kübler-Ross}
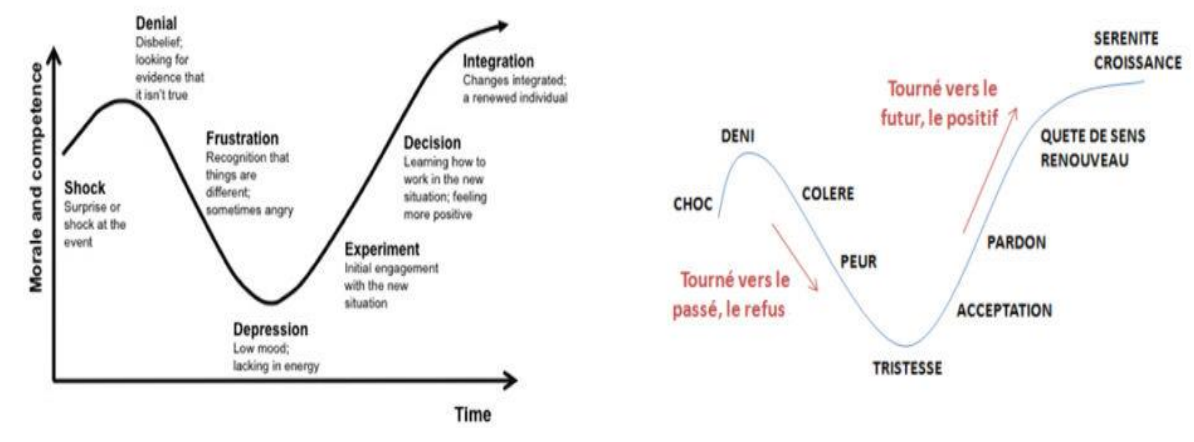

Figure 11 - La courbe de deuil (Kübler - Ross, 1969)

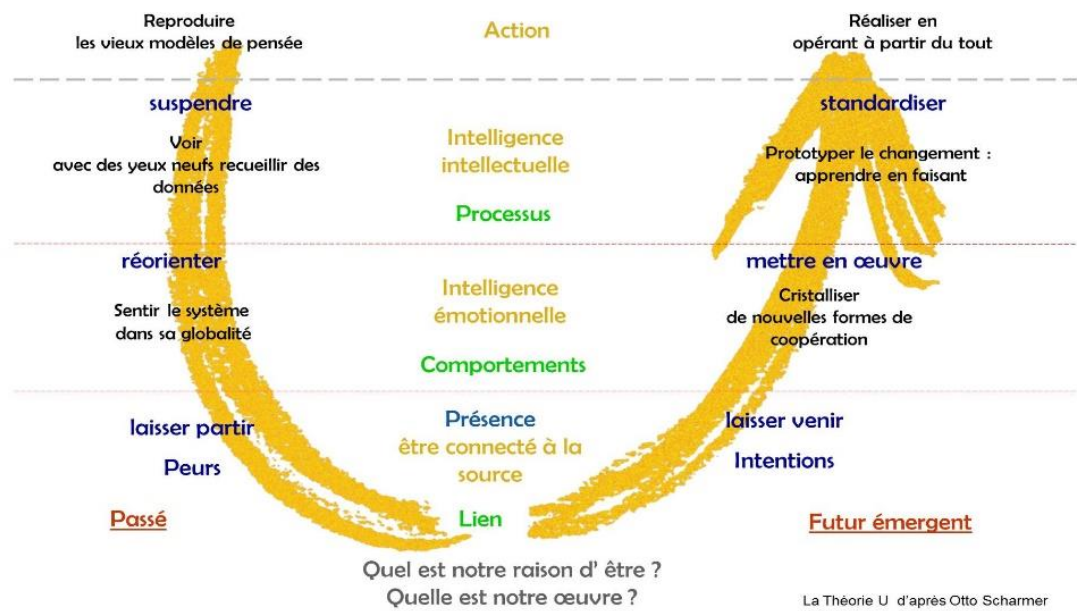

Figure 12 - Le processus en U, ou la Théorie U (Otto Scharmer, 2012)

Le point commun aux trois courbes est cette "positivisation théorique" de l'issue de la chute, du déclin, de l'implosion. En théorie, le système renait bien de ses cendres ici, et revient à son état initial de fonctionnement : c'est bel et bien le côté positif du dispositif ! 
Par contre, le nouveau modèle applicatif que cet article veut mettre en évidence prend en considération le fait que le système peut ne pas réussir à se redresser, à sortir de sa léthargie, de sa transition : il n'y arrive pas pour de multiples raisons ! Comment modéliser le système dans ce cas de figure ? Il va falloir créer un nouveau modèle applicatif.

En effet, arrivé à son point mort bas, dans sa phase de transition, le système de par ses aptitudes et compétences d'auto-organisation, va "re-naître" et exécuter ce que l'on va appeler sa résurgence, ou sa résilience. Du moins, c'est ce que les théories représentées par ces trois courbes laissent entendre !

Mais il se peut que le système après une tentative n'y arrive pas : il re-plonge, il n'arrive pas à "décrocher" ! C'est ce que la courbe en W (ou le processus en W) essaie de mettre en exergue. Et il se peut que l'on ne s'arrête pas à une seule tentative, à un seul essai, mais à plusieurs essais sans que l'on en détermine d'avance le nombre, disons tout simplement " $n$ tentatives" : d'où l'affectation de la lettre $\mathrm{W}$ à ce processus (qui prendrait la forme de cette lettre), et l'indice " $n$ " qui lui est juxtaposé, pour marquer l'itération probable de tentative de....résilience !

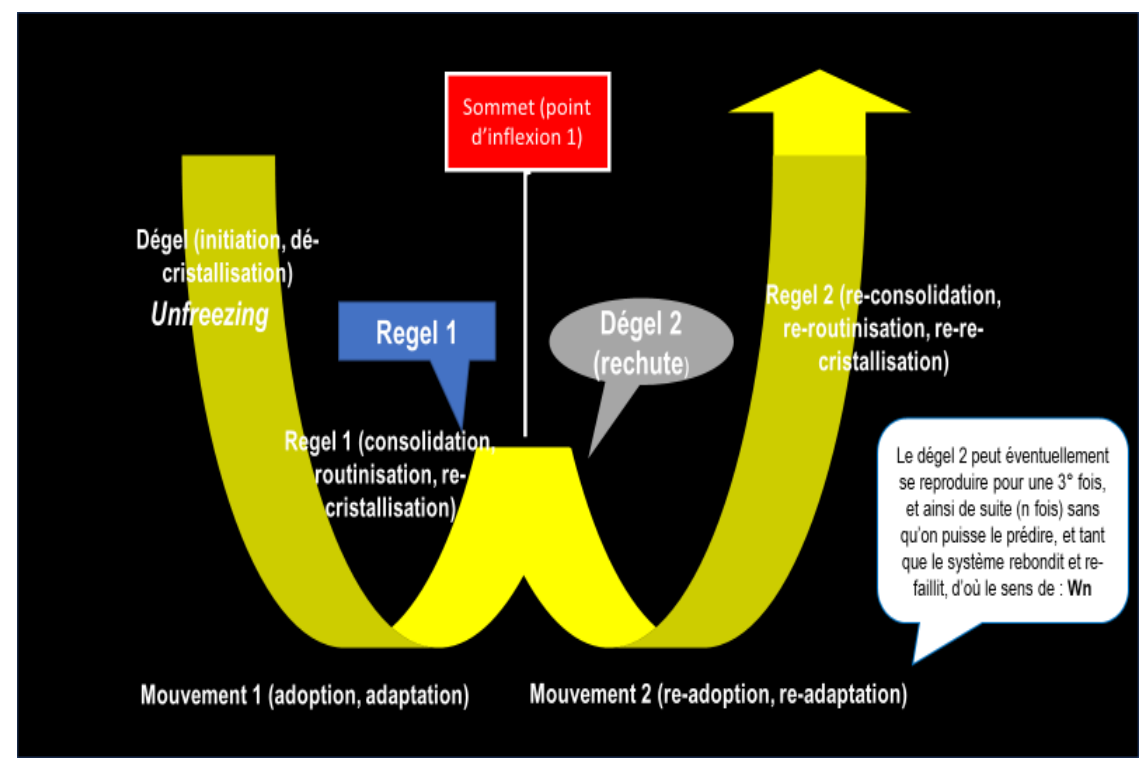

Figure 13 - Le Processus en W du changement. Réalisation : l'auteur.

\section{Le Processus en L}

Mais il est possible de pousser le raisonnement plus loin, cette fois-ci en s'aidant de la Loi de Murphy. En effet, selon cette loi, "tant qu'il y a un biais pour que cela aille mal, ca ira toujours dans ce sens" ! Un système affaibli, percuté (comme le STT) présenterait une brèche, une faille quelconque de par le principe de la néguentropie. La loi de Murphy s'énonce comme suit : "Tout ce qui est susceptible d'aller mal, ira mal", ou sous une autre formulation :"S'il existe au moins deux façons de faire quelque chose et qu'au moins l'une de ces façons peut entraîner une catastrophe, il se trouvera forcément quelqu'un quelque part pour entraîner cette voie".

Aussi, que les facteurs "murphyques" (de disruption) soient exogènes ou endogènes au système, pratiquement ce dernier semble être "voué et destiné" à mal tourner dans l'une des éventualités casuistiques de fonctionnement pré-citées. Serait-on alors en présence d'une "résonance morphique du système"?

\section{Résonance morphique}

Le mythe du célèbre Pont de Tacoma interpelle la doxa quant à la détermination causale de son effondrement. Le fait est là : le pont s'est effondré ! Peu importe les causalités mises en évidence, linéaires ou pas ? L'avis expert de l'architecte nous aidera à comprendre et à déterminer les causes possibles : le vent était de la partie provoquant une forte oscillation ayant mis à l'épreuve la résistance 
des matériaux qui sont entrés en résonance. Sans parler des militaires qui ont défilé en "pas cadencés" sur le Pont d'Angers en $1850 \ldots$

A la différence du Brexit (signifiant un système perturbé, secoué et télescopé par le futur départ du Royaume-Uni), après lequel l'Europe ne va pas disparaître, le Pont de Tacoma, lui, s'est réllement et effectivement "effondré, nécrosé et létalisé"! Quelle est la destinée d'un pont, serait-on tenté de poser comme question? Deux éventualités - dans une logique binaire - semblent se profiler : ou bien le pont tient bon, ou bien il s'effondre ! Il semblerait écrit quelque part dans une mémoire, qu'une voie est toute tracée pour les ponts : un pont ferait comme les autres ponts (cas d'effondrement)! L'hérétique Rupert Sheldrak ${ }^{10} \mathrm{e}$ a re-travaillé ce concept de "résonance morphique" en le transposant à un ou des domaines "élevés et transcendentaux" qu'on ne peut pas dès lors refuter! Une mémoire de l'univers" qui enregistrerait ce qu'ont fait ou subi les autres ponts antérieurement, et que ceux qui viendraient après emprunteraient...inévitablement selon cette "mémoire des choses".

Sheldrake (1989) définit la résonance morphique ${ }^{12}$ comme suit :

\begin{abstract}
C'est l'influence qu'exerce tout système auto-organisé passé sur les systèmes homologues présents. Atomes, molécules, cellules vivantes, plantes, animaux, sociétés, cultures, systèmes solaires, galaxies, sont des systèmes auto-organisés. Nos machines n'en sont pas, mais nos comportements ou nos pensées en sont. Chaque système se présente sous une certaine forme. La résonance morphique suppose que cette forme est comme mémorisée quelque part, dans un "champ morphique", ou "champ de forme". Prenez des pratiques nouvelles telles que le skate-board ou la navigation sur Internet : plus leurs adeptes sont nombreux, plus leurs champs de forme se renforcent et plus ces pratique deviennent faciles à mettre en oeuvre.
\end{abstract}

La loi de Murphy et le Pont de Tacoma avec la résonance morphique de Sheldrake dessinent ce que nous appelons "le Processus en L" (eu égard à la forme de la lettre en majuscule que le changement prendrait) : le système a failli ! En le rattachant au Tableau de Jones (qui a séquentialisé en 5 étapes les activités gouvernementales d'élaboration et de conception d'une politique publique), le Processus en L retrace une PPP (politique-programme-projet) qui n'a pas abouti, bref une politique publique qui disparait en queue de poisson, ou encore un système complexe qui se désintègre...

\title{
Processus en $L$ du changement
}

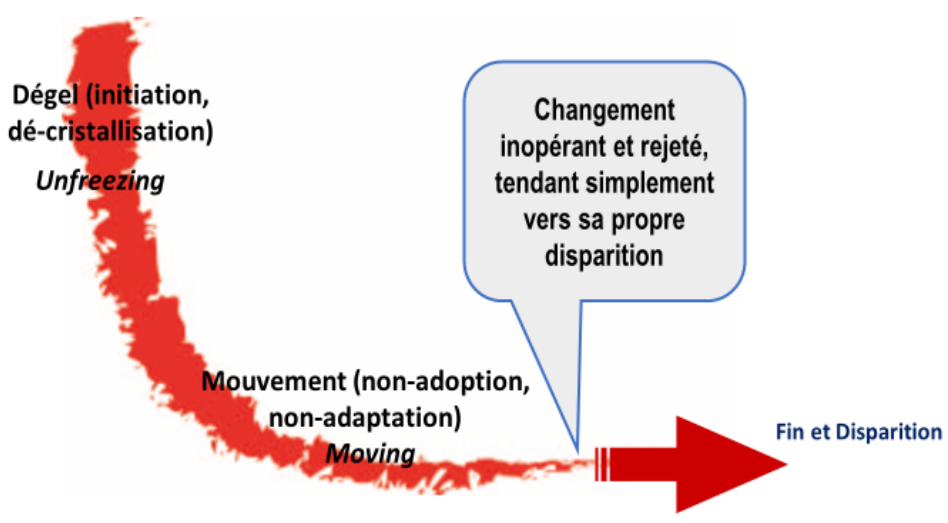

Figure 14 - Le Processus en L du changement. Réalisation : l'auteur.

\footnotetext{
${ }^{10}$ Rupert Sheldrake était interviewé à ce propos, selon l'article in Revue Acropolis «Rupert Sheldrake, un hérétique des temps modernes ».

${ }^{11}$ Lire à ce sujet la parabole du « centième singe » et celle des billes roulant sur une surface plane légèrement inclinée, selon le principe de chréode (qui stipule qu'une fois un phénomène apparu : plus il se produit, plus il est probable qu'il se reproduise).

${ }^{12}$ Interview de Rupert Sheldrake, disponible à l'adresse www.olivier-lockert.com/2013/11/25/interview-de-rupert-sheldrake/
} 


\title{
QUAND LE SYSTEME DEVIENT RESILIENT ET AGILE
}

\author{
Retour d'expérience
}

\section{Transition et Changement}

La dyade "transition et changement" constitue le point de départ de toute velleité auto-poïétique du système. En effet, au-delà de ses aptitudes d'auto-organisation, le (simple) système - à travers tout changement et toute transition - peut basculer vers l'auto-poïèse, cette "propriété d'un système de se produire lui-même, en permanence et en interaction avec son environnement, et ainsi de maintenir son organisation (structure) malgré son changement de composants (matériau)"(Angèle Kremer-Marietti, 2012).

La définition que Francesco Varela livre de l'autopoïèse se présente comme suit : "Un système autopoḯtique est organisé comme un réseau de processus de production de composants qui (i) régenèrent continuellement par leurs transformations et leurs interactions le réseau qui les a produits, et qui (ii) constituent le système en tant qu'unité concrète dans l'espace où il existe, en spécifiant le domaine topologique où il se réalise comme réseau. Il s'ensuit qu'une machine autopoïétique engendre et spécifie continuellement sa propre organisation. Elle accomplit ce processus incessant de remplacement de ses composants, parce qu'ele est continuellement soumise à des perturbations externes, et constamment forcée de compenser ces perturbations. Ainsi, une machine autopoïétique est un système à relations stables don't l'invariant fondamental est sa propre organisation (le réseau de relations qui la définit) (Varela, 1971, p.45).

Si le concept d'autopoïèse n'est pas du tout assimilable à une génération ou à une re-génération consécutive à un burn out, elle tend cependant vers la résilience, mais sous d'autres formes et avec d'autres paramètres.

\section{Résilience systémique et Résistance}

Initialement, la résilience désigne la capacité d'un matériau à encaisser les chocs, le "fait de rebondir". Ce mot vient du latin resilientia, de resiliens : la capacité d'un corps, d'un organisme, d'une espèce, d'un système à surmonter une altération de son environnement. Mais elle a évolué avec le temps et l'espace.

Si la « résilience» est, en sciences physiques, la capacité de résister aux chocs, ce terme désigne en psychologie la capacité de se construire alors que l'on a subi des traumatismes graves (Boris Cyrulnik, 2009). Et c'est le cas du système télescopé et traumatisé : de par son aptitude d'auto-organisation et auto poïétique, le système peut se relever, se régénérer peut-être pas à l'identique mais en mieux car prenant leçon du passé !

Observée et utilisée dans plusieurs domaines (psychologie, gestion d'entreprises, géographie, économie, physique, etc.), la notion de résilience est ici traitée et appliquée au système. Revenant au choc élastique et inélastique mentionné supra, il est utile de rappeler que le choc est absorbé par le système dès que l'on parle de résilience. Lors de la collision du système avec la crise venant de l'environnement, il n'y a pas eu refus ni rejet de ce choc par le système : au contraire, il l'a « accepté en le subissant »! En est-il « létalisé » pour autant?

Comme les deux faces de Janus, la résilience peut être également individuelle et organisationnelle. Cette fausse gémellité du concept - sans être un handicap - peut être exploitée car interdépendante : les éléments du tout impactent le tout, comme le tout impacte aussi les éléments constitutifs, en vertu du principe hologrammatique ${ }^{13}$ énoncé par Morin (1986). Dans cet ordre d'idées, le concept de résilience systémique comprenant d'une manière dyadique la résilience individuelle et celle organisationnelle est plus expressive et s'adapte mieux à l'esprit de l'article.

D'abord, parlant de la résilience individuelle, «la résilience de l'individu n'est pas une qualité innée que l'on possède ou non à la naissance. Elle se formera (éventuellement) plutôt avec le temps et grâce

\footnotetext{
${ }^{13}$ Il signifie que dans un système, dans un monde complexe, non seulement une partie se trouve dans le tout [...], mais le tout se trouve dans la partie. Non seulement, l'individu est dans une société mais la société est à l'intérieur de lui puisque dès sa naissance, elle lui a inculqué le langage, la culture, ses prohibitions, ses normes [...].
} 
à son environnement familial et social : «il s'agit d'un processus relatif qui n'est ni absolu ni stable : personne $n$ 'est résilient à toutes les variétés de traumatismes et personne n'est même assuré d'être toujours résilient à une forme de traumatisme qu'il a jusque-là surmonté avec succès. » (Tisserond, S., 2008).

Quant à la résilience organisationnelle, «c'est la capacité d'un groupe à éviter des chocs organisationnels en construisant des systèmes d'actions et d'interactions continus destinés à préserver les anticipations des différents individus les uns par rapports aux autres. » (Weick K.E., 2003).

Ainsi, la résilience systémique (ou la résilience d'un système) pour Hollnagel E. (2008) par exemple «c'est ce qui lui permet d'assurer sa sécurité au-delà de son imperméabilité aux perturbations. Le facteur humain dans le système d'organisation est primordial pour la résilience. L'individu ou le groupe est capable de percevoir les changements internes ou externes dans le système, de s'y adapter et de faire les réajustements nécessaires à la poursuite des fonctionnements. C'est, par contre, cet ajustement qui peut être mauvais et finalement détruire le système ». Cette combinaison gagnante du facteur humain et l'organisation (structurelle et fonctionnelle) confère au système cette potentialité de subir un traumatisme ou un changement brutal de sa structure, dans un premier temps, et au lieu de simplement résister et éventuellement se casser et se briser, de faire rebondir dans un deuxième temps le choc en l'absorbant, en l'assimilant pour revenir à son état initial, sinon à un état autrement différent, car déjà étant mieux préparé avec la possibilité d'anticipation ou de réaction.

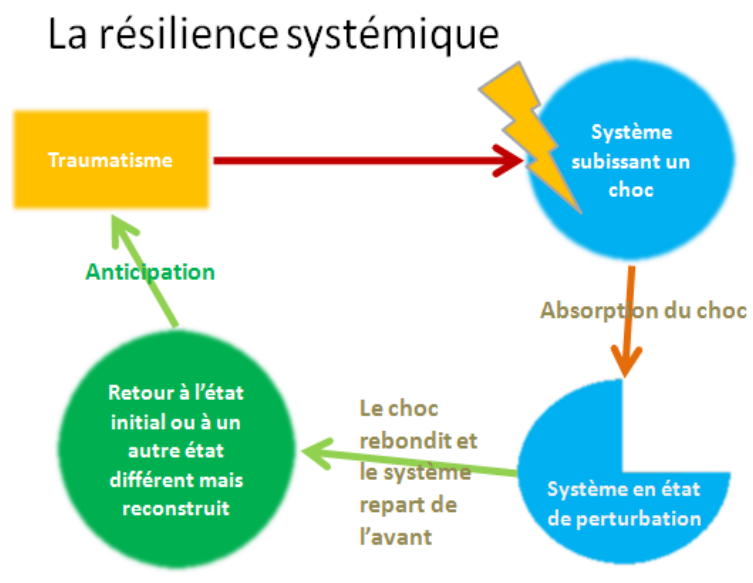

Figure 15 - La résilience systémique.

Source : http://nuagemotsclesferriercassezl3.unblog.fr/2016/04/29/resilience-definition.

La résilience sous-entend une capacité certaine de résistance du système. Point de départ de la résilience, la résistance est déjà inhérente au système, fait corps avec, mais c'est seulement un état, une faculté sous-jacente qu'il n'est besoin de créer ex nihilo mais qui se manifeste postérieurement à un évènement affectant - positivement ou négativement, mais la plupart du temps, négativement - le système. Une réaction plus qu'épidermique toutefois! Elle est synonyme d'opposition, de rejet, de fermeture à toute intrusion, à tout impact - pas nécessairement dense et vulnérant - affectant le système. Le mouvement des Gilets Jaunes ou la grève du 5 décembre 2019 impactant le système de retraite en France démontrent bien cet élan, ce mouvement de résistance !

\section{Agilité}

Après avoir résisté au choc, et assimilé le changement y connexe, et dans sa pleine résilience, qu'est-il attendu du système ? Comment il est censé se comporter après avoir survécu aux aléas ? La réponse à ces questions convergerait vers l'agilité : le système obtiendra et méritera une "valeur ajoutée" avec la méthode Agile. Un système agile en bref !

Par définition, une méthode Agile est une approche itérative et collaborative, capable de prendre en compte les besoins initiaux du client, des parties prenantes et ceux liés aux évolutions. 
Initialement rapportée dans les années 90 au domaine informatique pour des projets de conception, de production et d'amélioration des logiciels par rapport aux clients qui en ont exprimé la demande personnalisée d'acquisition, pourquoi ne pas essayer de la transposer à l'étude des systèmes ? Par exemple, en science politique en général, et en politiques publiques - systèmes complexes par excellence - en particulier!

"L'Agilité est l'habilité de créer et de répondre au changement dans le but d'avoir du succès dans un environnement d'affaires turbulent» (Highsmith, 2009). L'introduction de l'agilité dans la gestion de PPP (Politique-Programme-Projet) a complètement modifié la manière de l'appréhender en y injectant du mou, de la fluidité dans les processus. Très adaptative, la méthode se fond et s'intègre parfaitement dans le processus, et grâce aux itérations très brèves et courtes (sprint) avec les parties prenantes pour une amélioration continue, elle permet une gestion performante adoubée par une faculté d'anticipation et de réaction rapide. Les politiques publiques mises en œuvre par les autorités gouvernementales pourraient fonctionner de la sorte. Un système répondant à ces qualificatifs est ce qu'on appelle un système agile.

Une forte pléthore de méthodes agiles inonde actuellement le paysage organisationnel et institutionnel, et la plus en vogue est la Méthode Scrum. Cette dernière permet la réalisation de projets complexes en favorisant l'interaction (va-et-vient fréquent et rapide) avec les membres de l'équipe et les dirigeants, la collaboration avec les clients et la réactivité face aux changements, en bref avec et entre les parties prenantes !

Innovation managériale par excellence, par opposition à la démarche gauche et lourde de gestion et de conduite de projet classique, la méthode agile est un outil «léger et souple » permettant au système d'éviter en partie le choc attendu par anticipation sinon par réactivité. La faisabilité en a recours aux 3 piliers qui sont :

1. La transparence : un système opaque, sans communication entre les membres, avec un certain ostracisme communicationnel sélectif ou occulte, ne contribue point à rendre un processus fluide, car contenant des zones d'ombre ;

2. L'inspection : mal aimée et rejetée, cette pratique fournit un bilan régulier des résultats afin de pister les « gaps » auxquels il faudra apporter des corrections

3. L'adaptation : phase ultime découlant des corrections et qui permet de solutionner les écarts en « corrigeant le tir ».

\section{Les Avatars processuels de la pensée systémique}

(voir Figure 16 ci-contre)

\section{DISCUSSION - CONCLUSION}

Ce n'est pas parce qu'on a adopté un système agile que l'on est hors de danger, loin de là ! C'est nécessaire mais pas suffisant! Une issue probable à cette situation aporétique est (i) la complexification du système complexe et (ii) la latéralisation des politiques publiques !

En termes de complexité, la résilience du système post-transitionnel pratiquant l'agilité le conduit vers un attracteur et réduit considérablement sa vulnérabilité, ce qui le mènerait vers un état soutenable.

(i) Concernant la Complexification, il s'agit de pousser le système dans ses derniers retranchements afin de percevoir par quel(s) bout(s) le prendre pour trouver une solution aux problèmes du système. L'exemple typique le plus courant est la parabole de la « grève pour 5 Directrices » (voir encadré ${ }^{\circ} 1$ ) livrée par Jean-Louis Lemoigne $(1999$, p. 66) :

(ii) Quant à la latéralisation des politiques publiques (ou du système), elle est cette autre façon de raisonner, de voir et traiter le problème "en sortant du cadre », ce que l'Acta Europeana Systemica $\mathrm{n}^{\circ} 7$ a mis en évidence avec «Thinking Outside The Box». Métaphoriquement, avoir le nez dans le guidon ne permet pas de voir la route et d'apprécier l'environnement, pour un cycliste ! Prendre de la hauteur, de la distance permet de solutionner le problème du système, le problème du tout ! C'est comme si on demandait de relier 9 points avec 4 traits sans lever la main ni revenir deux fois sur un même point ! La solution est de sortir du cadre virtuel et théorique que nous nous sommes confectionné involontairement de par 
notre formation rationnelle cartésienne ! Quitte à trouver la solution au problème via la sérendipité !

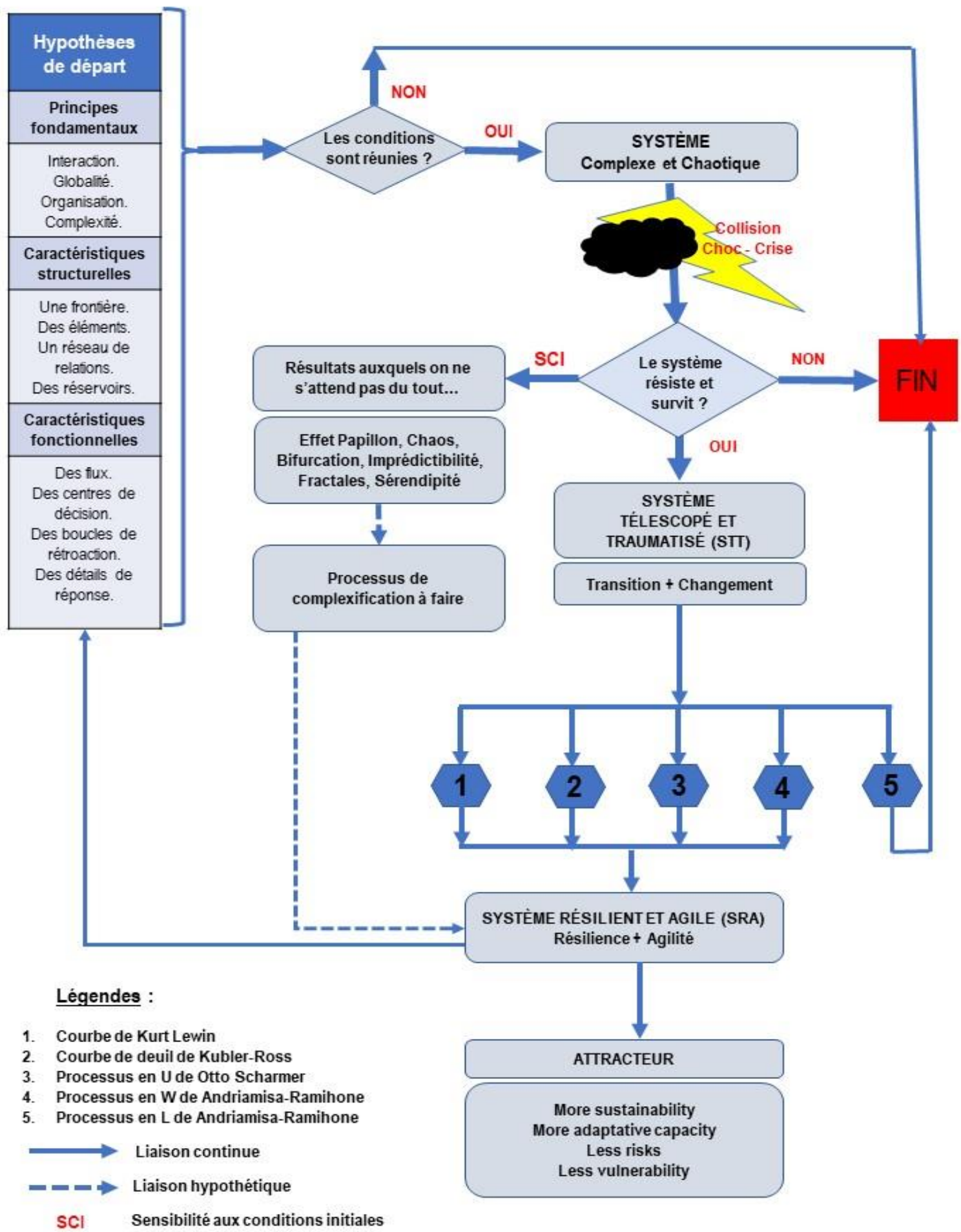

Figure 16 - Les avatars processuels de la pensée systémique transitionnelle. Réalisation : l'auteur 
Encadré $n^{\circ} 1$

La parabole de la grève pour cinq Directrices

Pour résoudre le conflit, complexifiez-le....

\begin{abstract}
Dans une petite banque américaine où la gent féminine représentait plus de $50 \%$ du personnel, la grève s'annonçait si, au moins parmi les dix directeurs (tous des hommes), 5 ne sont pas désormais des femmes ! Ce que la Direction exclusivement masculine a aussitôt refusé sans pouvoir justifier son refus (on ne peut plus légalement prétendre que les femmes, parce que femmes, sont moins capables que les hommes d'assurer des fonctions de direction dans une banque). Le problème se pose en termes de rationalité arithmétique, d'un côté et de tradition machiste, de l'autre : il faut que l'un des deux camps craque !

Jusqu'au jour où l'on proposait de reposer de façon complexe ce problème tenu pour simple : était-il vraiment mono-dimensionnel et mono-critère ? Ne pouvait-on convenir de modifier sa représentation, quitte à la complexifier, en y reconnaissant plusieurs niveaux concomitants et non un seul ? Est-ce que, par exemple, le pourcentage de femmes cadres n'était pas également important ? Et les commodités pratiques de travail dans les bureaux (horaires variables, cafétarias, acoustique, etc.). En reconnaissant une représentation multi-dimensionnelle et multi-niveaux du problème, en exprimant le caractère multicritère des projets de chacun et de l'organisation dans l'ensemble, ne créait-on pas un nouvel espace de négociation au sein duquel des solutions possibles, jusque-là complètement ignorées, pouvaient s'avérer pertinentes pour toutes les parties ? [...\} La complexification de la représentation suscitant des espaces de solutions nouvelles possibles conduisit à des négociations effectives dont les résultats furent tenus pour satisfaisants par chacun.
\end{abstract}

Source : La modélisation des systèmes complexes (Lemoigne, 1999, page 66)

\title{
REFERENCES
}

Autissier, D. et Moutot, J.M,. (2013). La boite à outils de la conduite du changement. Dunod, Paris Bertalanffy (v), L. (1962). Modern theories of development : An introduction to theoritical biology. Trad. Woodger, J. H., Harper Torchbook, New York.

Bertalanffy (v), L. (1993). Théorie générale des systèmes. Dunod, Paris.

Boussaguet, L. (dir), Jacquot, S. et Ravinet, P. Dictionnaire des politiques publiques. Les Presses de Sciences Po, Paris.

Bridges, W. (2009). Managing Transition - making the most of change. Third Edition, Da Capo Press, Cambridge.

Cannon, W. (1932). The wisdom of the body. Norton, New York.

Caublot, M., Poli, G., et Arnouil Deu, E. "Intérêts de l'approche écosystémique de la qualité de l'accueil des structures de la petite enfance pour la recherche et les pratiques professionnelles", in Revue Spirale 2014/2 (N70), pp. 137-150

Claeys, D. \& Minati, G. (2018). «A systems thinking approach of crises: From optimization to change strategy? ». Acta Europeana Systemica : Revue en ligne de l'Union Européenne de Systémique, volume ${ }^{\circ} 08 / 2018$, pp.1-2.

Crozier, M. et Friedberg, E. L'acteur et le système. Éditions du Seuil, Paris.

Cyrulnik, B. (2009). La Résilience : entretien avec Boris Cyrulnik (Français) Broché. Le Bord de l'Eau, Bordeaux.

Durand, D. (1979). La systémique. PUF, Paris.

François, C. (1998). «La systémique : un méta-langage connectif ». Revue Internationale de Systémique, vol.12, Nº4-5, pp. 405 à 418.

Godelier, Maurice (????). «L'objet et les enjeux ». Godelier, Maurice (dir.). Transitions et subordinations au capitalisme, p. 36-37.

Goldsmith, E. (1997). « Le chemin............ ». Interculture / Cahier $n^{\circ} 132$, volume XXX, $\mathrm{N}^{\circ} 1$

/HIVER-PRINTEMPS, pp. 11-17.

Highsmith, J. Agile Project Management. Addison Wesley Libri (rattachée à Pearson PLC), Londres.

Hollnagel, E. (2008). «Resilience-the Challenge of the unstable», dans Resilience Engineering. Concepts and Precepts. Ashgate 
Kemp, R., Loorbach, D., et Rotman, J. « Transition management as a model for managing processes of co-evolution towards sustainable development». The International Journal of Sustainable Development and World Ecology, North, 2004

Kremer-Marietti, A. "Réflexions sur l'autopoï̀se", in Revue de Philosophie et de sciences humaines DOGMA, octobre 2012.

Kübler-Ross, E. (1969), Les derniers instants de la vie, Labor et Fides, Genève (Suisse)

Lemoigne, J.L. (1999). La modélisation des systèmes complexes. Dunod, Paris

Loorbach, D. (2007). «Transition management : new mode of governance for sustainable

development ». North. 193

Moreau Defarges, P. (2008). L'ordre mondial. Armand Colin, Paris.

Morin, E. (1986). La méthode, T III - La connaissance de la connaissance. Éditions du Seuil, Paris.

Renier, J. (2018). «Crises systémiques : effondrement ? ou méta-morphose vers la Grande

Transition ? ». Revue en ligne de l'Union Européenne de Systémique AES, volume n 08 / 2018, pp. 285-300

Rosnay (de), J. (1975). Le macroscope. Éditions du Seuil, Paris.

Tisserond S., (2008). La résilience, Presses Universitaires de France - PUF, Paris.

Scharmer, O. C. (2012). Théorie U : Diriger à partir du futur émergent. Pearson, Londres.

Sheldrake, R. La mémoire de l'univers. Editions du Rocher, Monaco.

Varela, Francisco (1971). Autonomie et connaissance. Essai sur le vivant (1971), traduction en 1989 aux Éditions du Seuil.

Varela, F.G et. Maturana, F.H. De maquinas y seres vivos [en anglais "Autopoiesis : the organization of the living" (1973), in Autopoiesis and Cognition par Maturana H. et Varela F, Reidel 1980]

Waddington, C. H. (1975). The evolution of an evolutionist. Edinburgh University Press, Édimbourg.

Walliser, B. (1977). Systèmes et modèles. Éditions du Seuil, Paris.

Weick K.E. (2003). L'effondrement du sens dans les organisations. L'accident de Mann Gulch traduit par Laroche H. dans Le sens de l'action : Karl Weick : sociopsychologie de l'organisation, Vidaillet B., Vuibert. 
